Article

\title{
THz Spectroscopic Investigation of Wheat-Quality by Using Multi-Source Data Fusion
}

\author{
Hongyi Ge ${ }^{1,2,3}$, Yuying Jiang ${ }^{1,3}$ and Yuan Zhang ${ }^{1,3,4, *}$ \\ 1 Key Laboratory of Grain Information Processing \& Control, Ministry of Education, Henan University of \\ Technology, Zhengzhou 450001, China; gehongyi@haut.edu.cn (H.G.); jiangyuying11@163.com (Y.J.) \\ 2 College of Information Science and Engineering, Henan University of Technology, Zhengzhou 450001, China \\ 3 Key Laboratory of Henan Province for Grain Photoelectric Detection and Control, Zhengzhou 450001, China \\ 4 National Engineering Laboratory for Wheat \& Corn Further Processing, Zhengzhou 450001, China \\ * Correspondence: zy_haut@163.com; Tel.: +86-0371-6775-6610
}

Received: 4 October 2018; Accepted: 9 November 2018; Published: 14 November 2018

check for updates

\begin{abstract}
In order to improve the detection accuracy for the quality of wheat, a recognition method for wheat quality using the terahertz $(\mathrm{THz})$ spectrum and multi-source information fusion technology is proposed. Through a combination of the absorption and the refractive index spectra of samples of normal, germinated, moldy, and worm-eaten wheat, support vector machine (SVM) and Dempster-Shafer (DS) evidence theory with different kernel functions were used to establish a classification fusion model for the multiple optical indexes of wheat. The results showed that the recognition rate of the fusion model for wheat samples can be as high as $96 \%$. Furthermore, this approach was compared to the regression model based on single-spectrum analysis. The results indicate that the average recognition rates of fusion models for wheat can reach $90 \%$, and the recognition rate of the SVM radial basis function (SVM-RBF) fusion model can reach 97.5\%. The preliminary results indicated that THz-TDS combined with DS evidence theory analysis was suitable for the determination of the wheat quality with better detection accuracy.
\end{abstract}

Keywords: THz spectroscopy; Multi-Source Data Fusion; support vector machine; DS evidence theory; wheat quality

\section{Introduction}

Wheat is one of the major food sources in the world. It contains starch, protein, carbohydrates, lipids, and is a healthy food source of multiple nutrients and dietary fiber, particularly in many countries where wheat products are primary foods [1-4]. Inadequate control of the storage conditions can easily lead to mildew, moisture regain, germination, and insect infestation, which all reduce wheat quality $[5,6]$. Therefore, it is highly desirable to conduct research to obtain a fast and non-destructive detection method for wheat quality. Such a method could also effectively reduce post-harvest losses of grains and improves food safety.

Methods for inspecting quality of stored wheat as reported by domestic and foreign research include [7-10] chemical methods (measurement of major nutrient content, such as protein, carbohydrates, etc.), electronic nose, machine vision, spectrum detection (infrared spectroscopy). These methods are time-consuming and laborious, with a high consumption of both samples and reagents, and they have limited inspecting capabilities. THz radiation is sensitive to the vibrational states of the entire molecule, which means that terahertz radiation can be used to identify many materials. Due to its unique advantages such as the low energy of single photons and the "fingerprint character", THz time-domain spectroscopy (THz-TDS) is an effective non-contact measurement technique to analyze the internal composition of a substance. THz-TDS can also provide a direct 
measurement of the time-resolved amplitude and phase information, which are related to the absorptive and refractive properties of the sample, THz-TDS is currently the most used THz technology because it has important applications in the fields of biomedicine, material science, national defense, and quality control [11-15].

With regards to the qualitative and quantitative analysis of material using $\mathrm{THz}$ waves, many researchers have been focusing on the optical and spectral characteristics of the material within the $\mathrm{THz}$ band, and they carried out the measurement of the $\mathrm{THz}$ absorption coefficient and the refractive index coefficient, as well as an analysis of this single optical parameter. Many studies [16-22] have reported the combination of $\mathrm{THz}$ spectroscopy and chemometric methods (principal component analysis, partial least squares, support vector machines) to achieve a quantitative analysis of materials, and they achieved good results. The research group [23] used THz-TDS technology and the chemometric method to identify samples of different quality. The single-spectrum of wheat grain sample was used as the input to the constructed models, the comparison results demonstrated that the PCA-SVM approach can obtain satisfactory prediction accuracy for identifying the four types of wheat grain. However, the recognition rates for normal and germinated wheat were relatively high, while the recognition rates for moldy and worm-eaten wheat were low. The recognition rates for different models vary considerably.

This paper aimed to inspect different wheat qualities through the combination of multi-source information fusion technology and the THz-spectrum identification method in order to improve the recognition accuracy for wheat-quality classification. The wheat quality defects non-destructive detecting model used multi-source information fusion technology by fusing information of the absorption and refractive index spectrum of wheat samples of different quality. In the decision-making layer of fusion, the optical parameters were trained using an RBF kernel-function SVM, and the sub-classifier for wheat of varying quality was generated. The output results of the sub-classifier were fused using DS evidence theory. In the feature layer of fusion, the SVM method was used to construct a wheat-quality classification model using a feature layer, and the validation was carried out. Finally, the optimal fusion and classification methods of multi-source information for wheat quality were determined. These can be applied to identify and detect storage induced quality defects, and to provide the foundation for the application of $\mathrm{THz}$ technology for grain quality detection.

\section{Experimental Methods}

\subsection{Experimental Setup}

The spectra of the samples were measured using a Zomega-Z3 type THz-TDS system, which is located in the key laboratory grain information processing and control, Ministry of Education, Henan University of Technology (Zhengzhou, China). The detailed description of the device and measurement system are described in the literature [20,23]. A femtosecond fiber laser was used as light source, with a center wavelength of $780 \mathrm{~nm}$, a repetition rate of $80 \mathrm{MHz}$, and a pulse width of $100 \mathrm{fs}$. The related system parameters of the Z3-type THz-TDS are shown in Table 1, and the acquisition mode for the sample's spectral data was the transmission mode.

Table 1. System parameters of the Z3 THz-TDS (time domain spectroscopy).

\begin{tabular}{cc}
\hline Performance Index & Parameter Values \\
\hline Pump Source & Femtosecond fiber laser \\
Pumping capacity & $<10 \mathrm{~nJ}$ \\
Spectral range & $0.1-3.5 \mathrm{THz}$ \\
Frequency domain resolution & $<5 \mathrm{GHz}$ \\
Longest time delay & $1.3 \mathrm{~ns}$ \\
Dynamic range & $>70 \mathrm{~dB}$ (peak value) \\
THz radiation source & LT-GaAs photoconductive antenna \\
THz detector & ZnTe electro-optic crystal \\
\hline
\end{tabular}


All spectroscopic measurements of the wheat samples were performed at room temperature, 292 K. During the entire measurement, in order to reduce the effect of air moisture on the absorption of $\mathrm{THz}$ wave, the $\mathrm{THz}$ light path was filled with dry nitrogen. In addition, in order to reduce the measurement error, each sample was measured 5 times and the mean value was calculated.

\subsection{Sample Preparation and Parameter Extraction}

The preparation process of the wheat samples with different qualities was reported in the literature [23]. For sprouting wheat, the wheat samples were cleaned and soaked in water for $2 \mathrm{~h}$ and drained, and then enclosed in containers, and incubated at $25{ }^{\circ} \mathrm{C}$ for $18 \mathrm{~h}$; For moldy wheat, the wheat samples were moistened at a humidity of $28 \%$ and were put in a circular Petri dish, and then put into an incubator box at a constant temperature of $20^{\circ} \mathrm{C}$ for 2 days; For worm-eaten wheat, the nutrient component was partially eaten by corn elephants. The culture samples were used for measurement. The moisture content of the samples was about $12.5 \%$, measured by the grain moister sensor. The main component of wheat is measured, the protein content is $12.31 \%$ (moldy wheat), $10.22 \%$ (worm-eaten wheat), $14.97 \%$ (germinated wheat), and $15.43 \%$ (normal wheat) respectively, and the fat content is $5.01 \%$ (moldy wheat), 3.15\% (worm-eaten wheat), 5.27\% (germinated wheat), and 6.76\% (normal wheat) respectively. The average protein content is $13.23 \%$, the average fat content is $5.05 \%$, and the standard deviations of protein content and fat content are 0.024 and 0.015 . Wheat samples were ground into fine powder, which was subsequently sieved by filtering laws using 200-eye sieves; the wholemeal flour was used and then pressed into $1 \mathrm{~mm}$ thick pellets with a pressure of 10 tons for $5 \mathrm{~min}$, all the samples were labeled according to their properties as being worm-eaten, moldy, sprouting, or normal. When the sample was tested with the THz-TDS system, the THz pulse was absorbed and scattered by the sample. After a fast Fourier transform (FFT), the time domain signal of the sample was transformed into the frequency domain signal $[24,25]$.

$$
\widetilde{E}(\omega)=A(\omega) e^{-i \phi(\omega)}=\int d t E(t) e^{-i \omega t}
$$

Here, $A(\omega)$ is the amplitude ratio of the sample to reference signal, $\phi(\omega)$ is the phase difference between sample and reference signal, $\phi(\omega)=\phi_{s}(\omega)-\phi_{\text {ref }}(\omega)$. The absorption coefficient of sample $(\alpha)$ and refractive index coefficient $(n)$ can be calculated by comparing the sample spectrum with the reference spectrum.

$$
\begin{gathered}
\alpha=\frac{1}{d} \ln \frac{A_{r}}{A_{s}} \\
n=1+\frac{\left(\phi_{s}(\omega)-\phi_{r}(\omega)\right) C}{\omega d}
\end{gathered}
$$

where, $A_{r}$ and $A_{s}$ are the amplitudes of the reference signal and the sample signal, respectively. $\phi_{s}(\omega)$ and $\phi_{r}(\omega)$ are the phases of the sample signal and the reference signal, respectively. The variable $\omega$ is the frequency, $C$ is the speed of light, and $d$ is the thickness of the sample.

\subsection{The Multi-Source Information Fusion Method}

Multi-source information fusion is useful to analyze, control, and comprehensively process the multiple information resources for different times and space parameters, as well as to obtain new and more useful information to recognize and classify the measured object [26-28]. Multi-source information fusion can be divided into the following: data layer fusion, decision layer fusion, and feature layer fusion $[29,30]$. In this paper, the feature layer and the decision layer were used to fuse the spectral data.

Feature layer fusion extracts the feature vector from the collected original data (absorption coefficients and refractive index coefficients of the samples). It not only performs the compression of data and reduces the amount of interference data, but also maintains the important information 
contained in the original data. The pattern recognition method is then used for comprehensive analysis and treatment, which enables high recognition accuracy. The fusion process was shown in Figure 1.

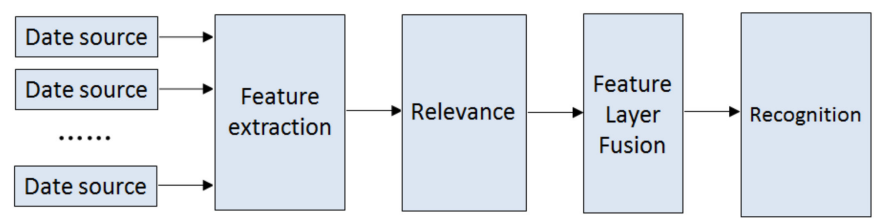

Figure 1. Feature layer information fusion.

SVM is a learning method based on statistical learning theory, which combines the advantages of non-linearity, high-dimensionality, and a good generalization performance. It not only improves the accuracy of the fusion results, but also improves the utilization efficiency of input model data greatly. This has become a popular topic of current research. The specific process of SVM is described in detail in the literature $[23,31,32]$.

Decision layer fusion serves to fuse the recognition results of multiple classification models for measurement targets, and it then executes the final reasoning and decision-making process [29]. Because the decision layer fusion performs the compression of the original data information, it is very flexible, requires a small amount of communication data, has a strong fault tolerance, and has a lower transmission bandwidth. In addition, it also has a relatively low requirement for the overall system. In this paper, Dempster-Shafer (DS) evidence theory method was used to create the decision-layer fusion model.

DS evidence theory [33-35] was first proposed by Dempster in 1967, and then expanded by Shafer and later further developed into DS evidence theory (Dempster/Shafer evidence theory). This method involves imprecise reasoning theory. It belongs to the field of artificial intelligence and has the ability to deal with uncertain information. When dealing with the problem of uncertainty including agnosticism, fuzziness, and randomness, the method with DS evidence combination at its core becomes very effective. DS evidence theory does not need to consider the sequence of multiple pieces of evidence, and can carry out appropriate adjustments according to the object properties during the fusion process. When the evidences are in conflict or in strong correlation, they can be grouped and respectively fused to reduce their impact. The fusion process is shown in Figure 2. The feature information of the sample was obtained, and then the support vector machine [36,37] using the radial basis kernel function was used to train the feature information. Then the corresponding sub-classifiers, RBF-SVM (absorption coefficient) and RBF-SVM (refractive index), were constructed. Finally, DS was used to fuse the output results of the sub-classifiers to obtain the final fusion results.

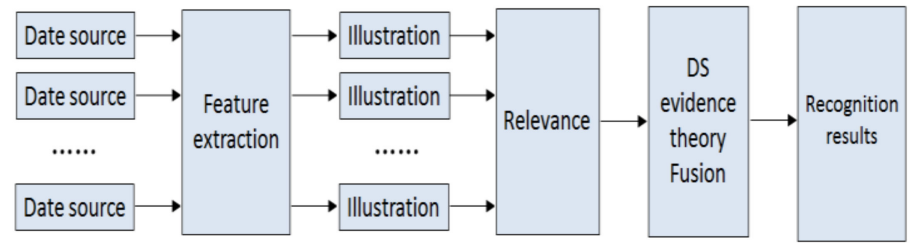

Figure 2. Decision-layer fusion.

\section{Results and Discussion}

\subsection{THz Spectrum Analysis of Wheat of Varying Quality}

Due to internal composition changes of moldy wheat, worm-eaten wheat, germinated wheat, and normal wheat, the interaction between molecules changes, and the vibration modes are different. In this case, the response characteristics are in the range of 0.2-1.6 THz. Four types of wheat samples were selected in this experiment, and 60 samples for each type were randomly selected for tests. The THz spectra of 240 wheat samples were measured using the THz-TDS. Each wheat sample was 
scanned 5 times, and the sample spectrum was the average of 5 scanning spectra in the range of 0.2-1.6 THz. The refractive index and absorption coefficient of the wheat samples are calculated from the $\mathrm{THz}$ spectra in the transmission mode, the validity and accuracy of all the measured spectra was evaluated by the standard deviations. Error bars that contain the mean and standard deviations for the 60 samples (one type) are shown in Figure 3. The mean values of the absorption spectra of moldy wheat, worm-eaten wheat, germinated wheat, and normal wheat are 2096.767, 2146.663, 2241.059, and 2466.321. The standard deviation ranges varies between 5.6344 and 72.5934 . The error bars of absorption spectra at $1.5 \mathrm{THz}$ with 71.2743 standard deviation and refractive index at $0.3 \mathrm{THz}$ with 0.19 standard deviation are more separated from others.
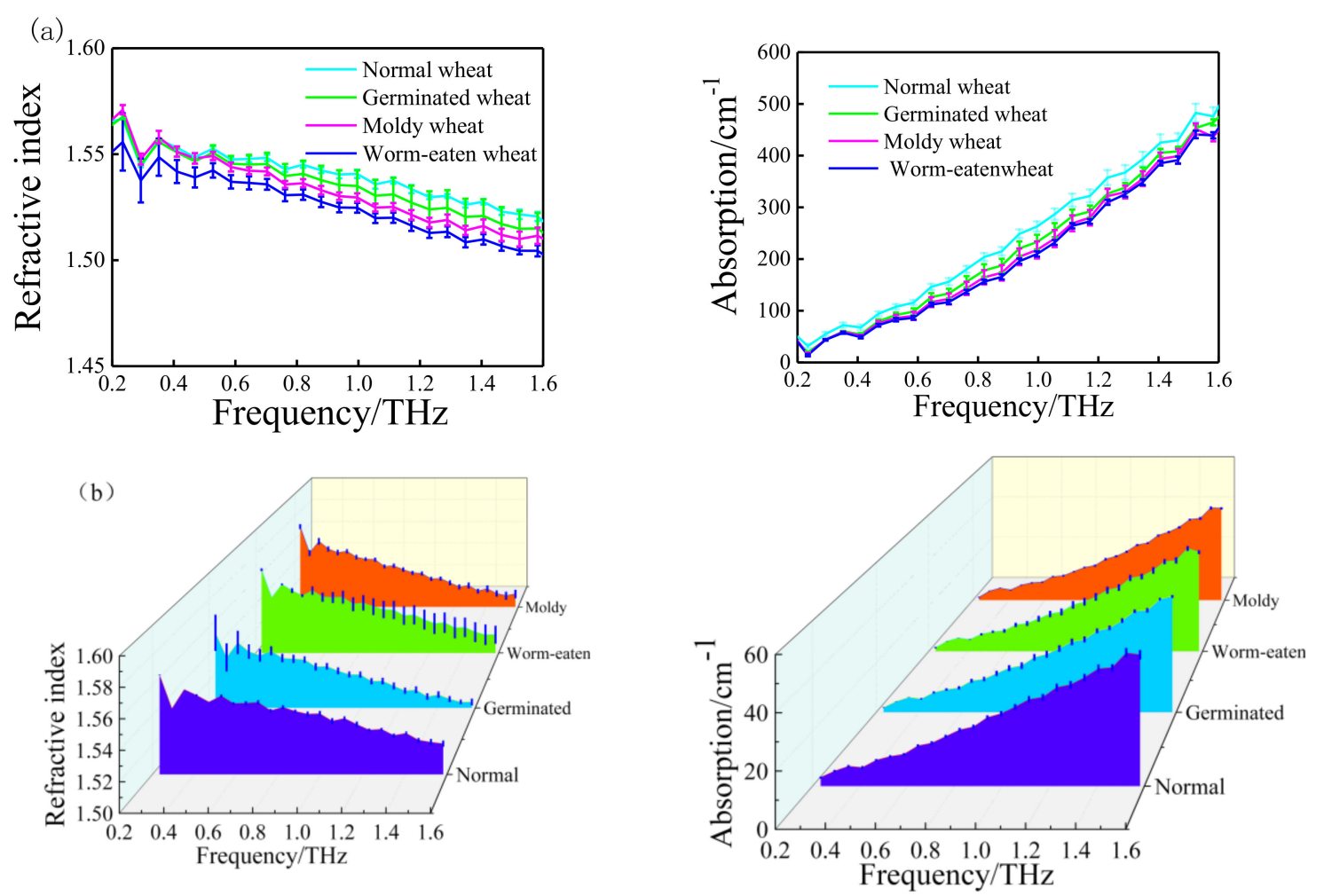

Figure 3. Refractive index spectra and absorption spectra (a) of different wheat samples and waterfall plot (b).

It can be seen from Figure 3 that the refractive index for the different wheat was 1.50-1.56, and the difference is very clear. The refractive index of normal wheat was the highest, and the order of intensity was $\mathrm{n}_{\text {worm-eaten }}$ wheat $<\mathrm{n}_{\text {moldy }}$ wheat $<\mathrm{n}_{\text {germinated }}$ wheat $<\mathrm{n}_{\text {normal }}$ wheat. The normal wheat sample had the most intense absorption and the highest absorption coefficient for $\mathrm{THz}$ waves, which is consistent with the analysis results in the literature [23]. The absorption curve of the sample did not have the obvious characteristic peak, and the differences in the absorption spectra for the wheat samples are not significant; chemometric methods were employed to investigate the relationship between the minor spectral differences and the measured wheat quality defects. In order to improve the detection accuracy for the quality of wheat, multi-source information fusion technology is proposed for the identification of wheat quality defects.

\subsection{The Wheat Classification Model Based on Feature Layer Fusion}

Principal component analysis (PCA) was used to extract the absorption spectrum and refractive index spectrum characteristics of wheat samples with varying quality. SVM was used as a model identification method, and different kernel functions were used to constructed different types of classification models. The main kernel functions were the linear kernel function, the polynomial 
kernel function, and the radial basis kernel function; they respectively were Linear-SVM, Poly-SVM, and RBF-SVM. The established models were compared to find the best feature layer fusion model.

(1) The establishment of the RBF-SVM fusion model

The calculated spectral information of 240 wheat samples was divided into two groups randomly according to the ratio of 2:1. Among them, the numbers of spectra in the training set and those in the test set were 160 and 80, respectively. The first eight principal component characteristics were fused into the absorption spectrum feature set of the wheat samples, and the first 10 principal components characteristics were fused into the refractive index spectra feature set of the wheat samples. The wheat classification and fusion model based on SVM was created. The RBF kernel function was chosen as the kernel function of the fusion model, and the optimal parameter () of the kernel function was calculated using the grid search optimization algorithm. Table 2 showed the recognition rate and the false judgment number for all types of wheat samples. After model optimization, the recognition rate of the training set of wheat samples with different quality reached $100 \%$, and the recognition rate for the test set was $97.5 \%$.

Table 2. Modeling results of the radial basis function support vector machine (RBF-SVM) wheat classification fusion model.

\begin{tabular}{|c|c|c|c|c|c|c|c|c|c|}
\hline \multicolumn{3}{|c|}{ Sample Type } & \multicolumn{4}{|c|}{ Determine Types } & \multirow{2}{*}{$\begin{array}{c}\text { False } \\
\text { Judgment } \\
\text { Number }\end{array}$} & \multirow{2}{*}{$\begin{array}{c}\text { Recognition } \\
\text { Rate of Each } \\
\text { Type (\%) }\end{array}$} & \multirow{2}{*}{$\begin{array}{c}\text { Overall } \\
\text { Recognition } \\
\text { Rate }(\%) \\
\end{array}$} \\
\hline & & & Normal & Germinated & Moldy & Worm-Eaten & & & \\
\hline \multirow{4}{*}{$\begin{array}{c}\text { Training } \\
\text { set }\end{array}$} & Normal & 38 & 38 & 0 & 0 & 0 & 0 & 100 & \multirow{4}{*}{100} \\
\hline & Germinated & 46 & 0 & 46 & 0 & 0 & 0 & 100 & \\
\hline & Moldy & 39 & 0 & 0 & 39 & 0 & 0 & 100 & \\
\hline & Worm-eaten & 37 & 0 & 0 & 0 & 37 & 0 & 100 & \\
\hline \multirow{4}{*}{ Test set } & Normal & 22 & 22 & 0 & 0 & 0 & 0 & 100 & \multirow{4}{*}{97.5} \\
\hline & Germinated & 14 & 0 & 14 & 0 & 0 & 0 & 100 & \\
\hline & Moldy & 21 & 0 & 0 & 20 & 1 & 1 & 95.24 & \\
\hline & Worm-eaten & 23 & 1 & 0 & 0 & 22 & 1 & 96.65 & \\
\hline
\end{tabular}

According to the table, the recognition rates of the established RBF-SVM fusion model for the normal, germinated, moldy, and worm-eaten wheat samples were $100 \%, 100 \%, 95.24 \%$, and $96.65 \%$, respectively.

\section{(2) The linear/poly-SVM fusion model}

Again, the first eight principal component characteristics were combined into the absorption spectrum feature set of the wheat samples. Then the first 10 principal component characteristics were combined into the refractive index spectra feature set of the wheat samples, and the different quality wheat classification and fusion model based on SVM was obtained. Linear and Poly kernel functions were chosen as the kernel function for the fusion model. Tables 3 and 4 showed the recognition rate and the false judgment number for all types of wheat samples. After model optimization, the recognition rate for the training set of wheat samples with different quality reached $100 \%$, and the recognition rates for the test set were $93.75 \%$ and $90 \%$, respectively.

Table 3. Modeling results of Linear SVM wheat classification fusion model.

\begin{tabular}{|c|c|c|c|c|c|c|c|c|c|}
\hline \multicolumn{3}{|c|}{ Sample Type } & \multicolumn{4}{|c|}{ Determine Types } & \multirow{2}{*}{$\begin{array}{c}\text { False } \\
\text { Judgment } \\
\text { Number }\end{array}$} & \multirow{2}{*}{$\begin{array}{c}\text { Recognition } \\
\text { Rate of Each } \\
\text { Type (\%) }\end{array}$} & \multirow{2}{*}{$\begin{array}{c}\text { Overall } \\
\text { Recognition } \\
\text { Rate (\%) }\end{array}$} \\
\hline & & & Normal & Germinated & Moldy & Worm-Eaten & & & \\
\hline \multirow{4}{*}{$\begin{array}{c}\text { Training } \\
\text { set }\end{array}$} & Normal & 38 & 38 & 0 & 0 & 0 & 0 & 100 & \multirow{4}{*}{100} \\
\hline & Germinated & 46 & 0 & 46 & 0 & 0 & 0 & 100 & \\
\hline & Moldy & 39 & 0 & 0 & 39 & 0 & 0 & 100 & \\
\hline & Worm-eaten & 37 & 0 & 0 & 0 & 37 & 0 & 100 & \\
\hline \multirow{4}{*}{ Test set } & Normal & 22 & 22 & 0 & 0 & 0 & 0 & 100 & \multirow{4}{*}{93.75} \\
\hline & Germinated & 14 & 0 & 13 & 0 & 1 & 1 & 92.86 & \\
\hline & Moldy & 21 & 1 & 0 & 19 & 1 & 2 & 90.48 & \\
\hline & Worm-eaten & 23 & 1 & 0 & 1 & 21 & 2 & 91.3 & \\
\hline
\end{tabular}


According to the table, the recognition rates for the established linear/poly-SVM fusion models for normal wheat samples reached $100 \%$. There was a difference between the recognition rates of germinated, moldy, and worm-eaten wheat samples. Furthermore, in the process of testing, there were two false judgments that falsely identified the moldy and worm-eaten wheat as normal wheat. The results showed that the recognition rate of the RBF-SVM fusion model for the four wheat samples is higher than for other models.

Table 4. Modeling results of Poly SVM wheat classification fusion model.

\begin{tabular}{|c|c|c|c|c|c|c|c|c|c|}
\hline \multicolumn{3}{|c|}{ Sample Type } & \multicolumn{4}{|c|}{ Determine Types } & \multirow{2}{*}{$\begin{array}{c}\text { False } \\
\text { Judgment } \\
\text { Number }\end{array}$} & \multirow{2}{*}{$\begin{array}{c}\text { Recognition } \\
\text { Rate of Each } \\
\text { Type (\%) }\end{array}$} & \multirow{2}{*}{$\begin{array}{c}\text { Overall } \\
\text { Recognition } \\
\text { Rate (\%) }\end{array}$} \\
\hline & & & Normal & Germinated & Moldy & Worm-Eaten & & & \\
\hline \multirow{4}{*}{$\begin{array}{c}\text { Training } \\
\text { set }\end{array}$} & Normal & 38 & 38 & 0 & 0 & 0 & 0 & 100 & \multirow{4}{*}{100} \\
\hline & Germinated & 46 & 0 & 46 & 0 & 0 & 0 & 100 & \\
\hline & Moldy & 39 & 0 & 0 & 39 & 0 & 0 & 100 & \\
\hline & Worm-eaten & 37 & 0 & 0 & 0 & 37 & 0 & 100 & \\
\hline \multirow{4}{*}{ Test set } & Normal & 22 & 22 & 0 & 0 & 0 & 0 & 100 & \multirow{4}{*}{90} \\
\hline & Germinated & 14 & 0 & 12 & 0 & 1 & 2 & 92.86 & \\
\hline & Moldy & 21 & 1 & 0 & 18 & 2 & 3 & 85.71 & \\
\hline & Worm-eaten & 23 & 1 & 0 & 2 & 20 & 3 & 86.96 & \\
\hline
\end{tabular}

\subsection{The Decision Layer Fusion Model for Wheat Recognition Using DS Evidence Theory}

The four wheat samples of normal wheat, germinated wheat, moldy wheat, and worm-eaten wheat were selected as study objects. Based on DS evidence theory, the classification and fusion model for wheat of different quality was established. The establishing process of the model was as follows:

(1) Respectively established the classification probability output of wheat corresponding to the absorption spectra and refractive index spectra for wheat of different quality.

For the absorption spectrum of different qualities of wheat, the first 10 principal components characteristics were combined into an absorption spectrum feature set, and the wheat classification model was established using the SVM pattern recognition method, with the optimal parameters of RBF kernel function, $\gamma$ and C, being 3.5 and 1.6. Then the probability outputs of the training set and the test set were obtained.

For the refractive index spectrum of different qualities of wheat, the first eight principal components characteristics were combined into spectrum feature set, and the wheat classification model was established using the SVM pattern recognition method with the optimal parameters of RBF kernel function, $\gamma$ and $C$, being 3 and 0.92 . Then the probability outputs of the training set and the test set for different qualities wheat were calculated.

\section{(2) Established DS evidence fusion rules}

The recognition framework of DS fusion model was assumed as $\Theta=\left\{\alpha_{1}, \alpha_{2}, \alpha_{3}, \alpha_{4}, U\right\}$. Among them, $\alpha_{1}, \alpha_{2}, \alpha_{3}, \alpha_{4}$ represents normal wheat, germinated wheat, moldy wheat, and worm-eaten wheat, $U$ is the wheat of uncertain quality, which may be any of the four types. Belabsorption and Belrefraction, respectively, are the belief functions of SVM classification model for the sample set with the same $\Theta$. Mabsorption and mrefraction are the basic probability assignment functions of the wheat sample absorption spectra and refractive index spectra SVM classifier for the training set of the four types of wheat samples of different quality. Mabsorption and Mrefraction are the basic probability assignments (BPA) of the absorption spectra and refractive index spectra SVM classifier for the test set of four different qualities. $M_{c}$ is the fusion probability function of the wheat samples absorption spectra and refractive index spectra SVM classifier using the fusion of DS evidence theory. The fusion rules are as follows:

$$
M_{\text {absorption }}=m_{\text {absorption }}(A) \operatorname{Bel}(A)
$$




$$
\begin{aligned}
& M_{\text {refraction }}=m_{\text {refraction }}(A) \operatorname{Bel}(A) \\
& K=\sum_{\substack{i=\text { absorption, } j=\text { refraction } \\
A_{i} \cap t_{j}=\phi}} M_{\text {absorption }}\left(A_{i}\right) M_{\text {refraction }}\left(A_{j}\right) \\
& M_{\mathcal{C}}(A)=\sum_{\substack{i=\text { absorption, } j=\text { refraction } \\
A_{i} \cap t_{j}=A}} M_{\text {absorption }}\left(A_{i}\right) M_{\text {refraction }}\left(A_{j}\right) /(1-K)
\end{aligned}
$$

Here, $A \in \Theta$. Decision rules: Set $\exists A_{1}, A_{2} \in \Theta$, and satisfy:

$$
M_{\mathcal{c}}\left(A_{1}\right)=\max \left\{M_{\mathcal{c}}\left(A_{i}\right), A_{i} \in \Theta\right\}, M_{\mathcal{c}}\left(A_{2}\right)=\max \left\{M_{\mathcal{c}}\left(A_{i}\right), A_{i} \in \Theta \cap A_{i} \neq A_{1}\right\}
$$

If it satisfies the conditions $\left\{\begin{array}{l}M_{\mathcal{c}}\left(A_{1}\right)-M_{\mathcal{c}}\left(A_{2}\right)>\varepsilon_{1} \\ M_{\mathcal{c}}(\Theta)<\varepsilon_{2} \\ M_{\mathcal{c}}\left(A_{1}\right)>M_{\mathcal{c}}(\Theta)\end{array}\right.$, among which $\varepsilon_{1}$ and $\varepsilon_{2}$ are the threshold of judgment, $A_{1}$ can be determined as the final decision.

(3) Recognition results of the classification fusion model for wheat using DS evidence theory

Following the above theory, normal, germinated, moldy, and worm-eaten wheat were selected as study objects in this paper, and through a combination of the absorption and refractive index spectra of the wheat samples, the classification fusion model for wheat quality defects was established using DS evidence theory. The recognition rate of the four wheat samples reached $100 \%$ in the training set,

\begin{tabular}{|c|c|c|c|c|c|c|c|c|c|}
\hline \multirow{2}{*}{\multicolumn{3}{|c|}{ Sample Type }} & \multicolumn{4}{|c|}{ Determine Types } & \multirow{2}{*}{$\begin{array}{c}\text { False } \\
\text { Judgment } \\
\text { Number }\end{array}$} & \multirow{2}{*}{$\begin{array}{c}\text { Recognition } \\
\text { Rate of Each } \\
\text { Type (\%) }\end{array}$} & \multirow{2}{*}{$\begin{array}{c}\text { Overall } \\
\text { Recognition } \\
\text { Rate (\%) }\end{array}$} \\
\hline & & & Normal & Germinated & Moldy & Worm-Eaten & & & \\
\hline \multirow{4}{*}{$\begin{array}{c}\text { Training } \\
\text { set }\end{array}$} & Normal & 38 & 38 & 0 & 0 & 0 & 0 & 100 & \multirow{4}{*}{100} \\
\hline & Germinated & 46 & 0 & 46 & 0 & 0 & 0 & 100 & \\
\hline & Moldy & 39 & 0 & 0 & 39 & 0 & 0 & 100 & \\
\hline & Worm-eaten & 37 & 0 & 0 & 0 & 37 & 0 & 100 & \\
\hline \multirow{4}{*}{ Test set } & Normal & 22 & 22 & 0 & 0 & 0 & 0 & 100 & \multirow{4}{*}{96.25} \\
\hline & Germinated & 14 & 0 & 14 & 0 & 0 & 0 & 100 & \\
\hline & Moldy & 21 & 0 & 0 & 20 & 1 & 1 & 95.24 & \\
\hline & Worm-eaten & 23 & 0 & 1 & 1 & 21 & 2 & 91.3 & \\
\hline
\end{tabular}
and the recognition rate of the test set was relatively low, $96.25 \%$. The recognition rate and the number of false judgment of different wheat samples are shown in Table 5.

Table 5. The result of the decision fusion model using the DS wheat classification model.

According to the table, the recognition rate of the DS evidence theory fusion model for all types of wheat samples in the training set was high, up to $100 \%$. For the test set, the recognition rates for normal wheat and germinated wheat were 100\%, while the recognition rates of the moldy and the worm-eaten wheat were relatively low, only $95.24 \%$ and $91.3 \%$, respectively. The experimental results indicate that, compared with the recognition rate obtained from the models based only on the absorption spectra or the refractive index spectra, the recognition rate using the DS evidence theory fusion model for wheat of different quality had improved. This is true especially for moldy and worm-eaten wheat samples, and the sample recognition rate has improved significantly.

\subsection{Comparison of the Different Fusion Models}

For the absorption and refractive index spectra of the four types of wheat, normal wheat, germinated wheat, moldy wheat, and worm-eaten wheat, the classification detection fusion model of 
multiple-spectral indices of wheat was established using DS evidence theory and SVM model with different kernel functions. The results show that the recognition rates of the fusion models are similar, and the prediction accuracies of the fusion models are higher than with the models established using the simple spectrum. In addition, the fusion models were able to accurately recognize the normal wheat samples. The main difference appeared in the germinated, moldy, and worm-eaten wheat samples, where the recognition rate for the fusion model established on RBF-SVM was slightly higher than that using DS evidence theory fusion.

For the PCA-SVM model established with the absorption spectrum only, the recognition rates for normal, germinated, moldy, worm-eaten wheat, and the overall recognition rate were $100 \%, 100 \%$, $85.71 \%, 82.61 \%$, and $92.08 \%$ respectively. For the PCA-SVM model established with the refractive index spectrum, the recognition rates for normal, germinated, moldy, worm-eaten wheat, and the overall recognition rate were $100 \%, 80.95 \%, 86.96 \%$, and $91.98 \%$, respectively. However, the corresponding recognition rates based on fusion models reached up to $90 \%$. The experiments indicate that, for wheat samples of different quality, the multi-source information fusion, established through a combination of the absorption spectrum and the refractive index spectrum, improves the recognition rate of wheat. More specifically, the recognition rate using the RBF-SVM feature fusion is the highest, suggesting it is the optimal method of multi-source information fusion. In order to further demonstrate the validity of the proposed method, the obtained results were examined in terms of the measurement time. Each sample was measured five times to generate an average spectrum, where the scan time was no more than five s. Thus, the prediction time for each sample is no more than six s (including the total scan time and the calculation time of one second) on a standard PC with four GB of RAM and a Pentium CPU. The classification recognition results for each sample can be completed within six s, indicating the feasibility of the fast information fusion methods.

\subsection{Discussion}

Chua et al. [38] uses the THz-TDS to measure absorption spectra of wheat grain with $8 \%, 12 \%$, and $18 \%$ moisture content, it also analyzes the relationship between the moisture content and $\mathrm{THz}$ transmission signals. It is well known in $\mathrm{THz}$ frequency region that liquid water presents a strong absorption. The absorption spectra of wheat grain of varying moisture content levels (dry, $12 \%, 12.5 \%$, and $13 \%$ ) were calculated using THz-TDS, which associated with both free water molecules and hydration water within the grain. In order to isolate the effect of the moisture content, the spectra were subtracted from the dry wheat grain. The absorption spectra were shown in the Figure 4 . It demonstrates that the samples have no obvious absorption peaks in the frequency range, and also shows that the moisture content was about $12.5 \%$, and has little influence on the $\mathrm{THz}$ radiation. The differences in $\mathrm{THz}$ absorption for different varieties of wheat are due to the variations in the composition of different wheat samples. Guo et al. [39] reports a method of evaluation of wheat seeds by using $\mathrm{THz}$ imaging mainly based on the reflection mode. However, few studies report multi-source information fusion methods on the multiple optical indexes of sample for the improvement in model performance. The applicability of THz-TDS combined with multi-source information fusion technology to determine the quality of wheat grain is present in this study. Better prediction results obtained were comparable with previous reports in the region of $0.2-1.6 \mathrm{THz}$. In particular, it is of great concern for agricultural products and food safety, particularly packaged goods. THz wave has the ability to penetrate a wide range of materials (such as paper, plastic, and cloth). Given this characteristic, we will continue to explore the quality of packaged wheat and examined the effect of packaging materials by using THz-TDS in the future. 
(a)

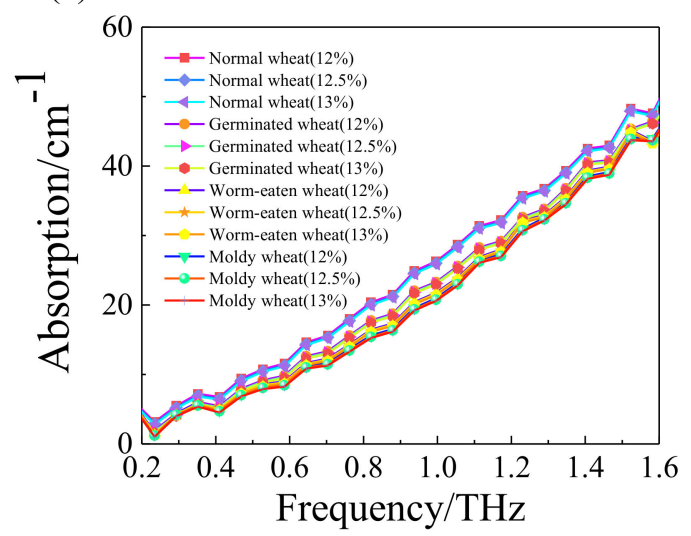

(c)

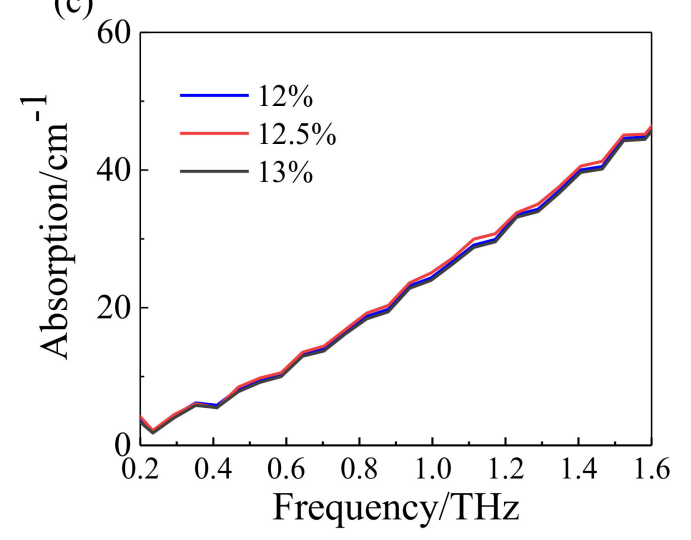

(b)
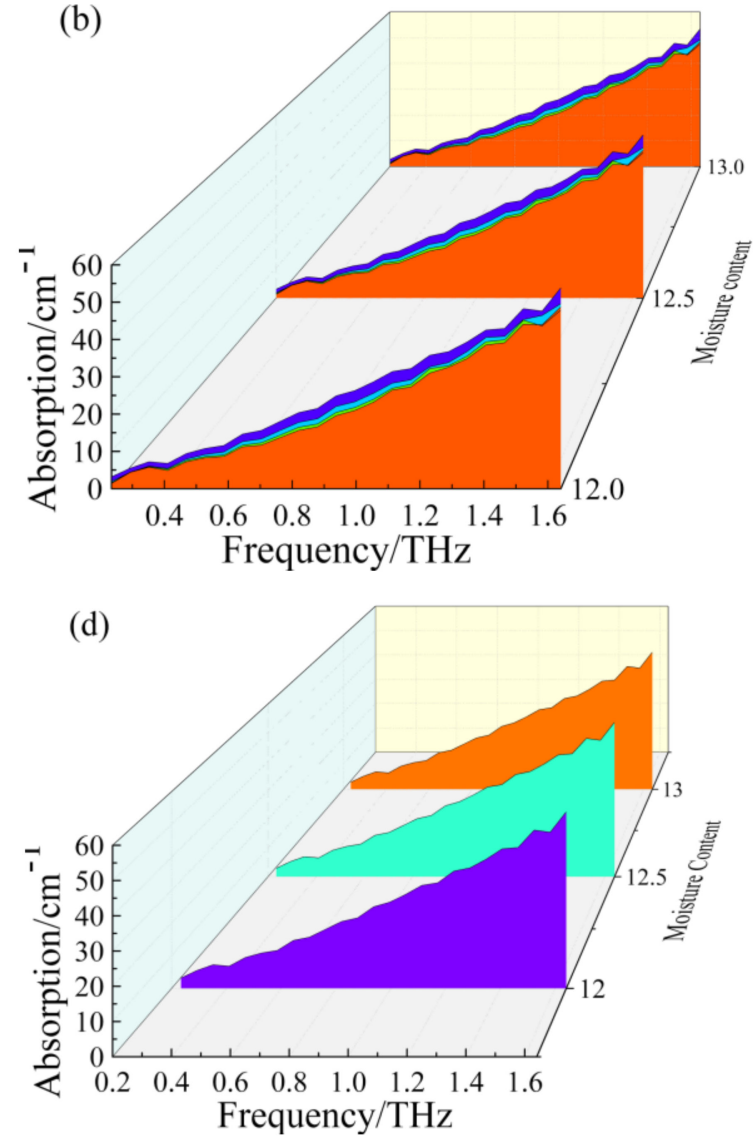

Figure 4. THz Absorption spectra of varying moisture content levels of different wheat grain (a) and waterfall plot (b) THz Absorption spectra of varying moisture content levels of worm-eaten wheat (c) and waterfall plot $(\mathbf{d})$.

In addition, the absorption spectrum was used in the literature [23], although a satisfactory result can be obtained by the constructed model. The fusion optical parameter (the absorption spectrum and the refractive index spectrum) provides rich information based on the molecular structure. In our work, these preliminary results indicated that THz-TDS combined with DS evidence theory analysis was suitable for the determination of the storage induced quality defects with better detection accuracy. However, the influencing factors of the spectra (such as plant areas, etc.), will need to be considered in further studies. Further research (including the development of a cost-effective $\mathrm{THz}$ spectrometer with high SNR and assay) is required to improve the robustness of the prediction model for real application in the agricultural products/food. Furthermore, the determination of lower levels of the defective samples would be of great value, as well as the further quantitative analysis of wheat grains of different germination times and stages of mold growth, and studies in which the defective samples are blended with the control wheat at ratios from 1:9 through to 9:1 in order to build to more valuable models to discriminate varieties.

\section{Conclusions}

In this paper, samples of normal, germinated, moldy, and worm-eaten wheat were investigated. Through combination of the absorption and the refractive index spectrum, SVM and DS evidence theory with different kernel functions were used to establish a classification fusion model for the multiple optical indexes of wheat. Our modeling results indicate that the recognition rates of fusion models for wheat can reach $90 \%$, and the recognition rate of the RBF-SVM fusion model can reach $97.5 \%$. To verify the validity of the fusion model, the model was compared with the PCA-SVM single-spectrum analysis regression model as reported in the literature. The result shows that the 
fusion model has a better prediction effect with respect to the recognition rate for wheat samples of different quality. The results indicate that the fusion model improves the detection accuracy for several indexes of wheat quality defects. Among the investigated fusion models, the RBF-SVM fusion model has the highest detection accuracy; therefore, it is the currently best multi-source information fusion method. The multi-source information fusion model of the THz spectrum can solve the recognition problem for wheat quality, which is of great significance for grain quality detection.

Author Contributions: H.G. and Y.J. conducted the algorithm design, experiments and analysis. Technical discussion and data processing were performed by H.G. Y.Z. has supervised the whole work. All authors contributed to the writing and the revision of the manuscript.

Funding: This work was supported in part by National Key Research and Development Program of China (No. 2017YFD0401003), in part by National High-tech R\&D Program of China (863 Program) (No. 2012AA101608), in part by Key Scientific Research Project of Henan universities of China(No. 16A510002), in part by the Doctoral Funds of the Henan University of Technology (No. 2015BS015), in part by the National Natural Science Foundation of China(No. 61705061, U1404617), in part by the Science and Technology Innovation Teams of Henan Universities (No. 16IRTSHN026), and in part by the Scientific Research Funds of Henan University of Technology (No. 2016QNJH30).

Conflicts of Interest: The authors declare no conflict of interest.

\section{References}

1. Prinsen, P.; Gutierrez, A.; Faulds, C.B.; del Rio, J.C. Comprehensive Study of Valuable Lipophilic Phytochemicals in Wheat Bran. J. Agric. Food Chem. 2014, 62, 1664-1673. [CrossRef] [PubMed]

2. Valipour, M. Land use policy and agricultural water management of the previous half of century in Africa. Appl. Water Sci. 2015, 5, 367-395. [CrossRef]

3. Yannopoulos, S.I.; Lyberatos, G.; Theodossiou, N.; Li, W.; Valipour, M.; Tamburrino, A.; Angelakis, A.N. Evolution of Water Lifting Devices (Pumps) over the Centuries Worldwide. Water 2015, 7, 5031-5060. [CrossRef]

4. Valipour, M. Global experience on irrigation management under different scenarios. J. Water Land Dev. 2017, 32, 95-102. [CrossRef]

5. Oviedo, M.S.; Ramirez, M.L.; Barros, G.G.; Chulze, S.N. Influence of water activity and temperature on growth and mycotoxin production by Alternaria alternata on irradiated soya beans. Int. J. Food Microbiol. 2011, 149, 127-132. [CrossRef] [PubMed]

6. Neethirajan, S.; Karunakaran, C.; Jayas, D.S.; White, N.D.G. Detection techniques for stored-product insects in grain. Food Control. 2007, 18, 157-162. [CrossRef]

7. McMullin, D.; Mizaikoff, B.; Krska, R. Advancements in IR spectroscopic approaches for the determination of fungal derived contaminations in food crops. Anal. Bioanal. Chem. 2015, 407, 653-660. [CrossRef] [PubMed]

8. Jaillais, B.; Roumet, P.; Pinson-Gadais, L.; Bertrand, D. Detection of Fusarium head blight contamination in wheat kernels by multivariate imaging. Food Control. 2015, 54, 250-258. [CrossRef]

9. Niu, Y.H.; Hua, L.; Hardy, G.; Agarwal, M.; Ren, Y.L. Analysis of volatiles from stored wheat and Rhyzopertha dominica (F.) with solid phase microextraction-gas chromatography mass spectrometry. J. Sci. Food Agric. 2016, 96, 1697-1703. [CrossRef] [PubMed]

10. Neethirajan, S. Jayas, D.S.; White, N.D.G. Detection of sprouted wheat kernels using soft X-ray image analysis. J. Food Eng. 2007, 81, 509-513. [CrossRef]

11. Ferguson, B.; Zhang, X.C. Materials for terahertz science and technology. Nat. Mater. 2002, 1, $26-33$. [CrossRef] [PubMed]

12. Siegel, P.H. Terahertz technology in biology and medicine. IEEE Trans. Microw. Theory 2004, 52, $2438-2447$. [CrossRef]

13. Shimada, Y.; Iida, H.; Kinoshita, M. Recent Research Trends of Terahertz Measurement Standards. IEEE Trans. Terahertz Sci. Technol. 2015, 5, 1166-1172.

14. Xie, L.J.; Gao, W.L.; Shu, J.; Ying, Y.B.; Kono, J.C. Extraordinary sensitivity enhancement by metasurfaces in terahertz detection of antibiotics. Sci. Rep. 2015, 5, 8671. [CrossRef] [PubMed] 
15. Gowen, A.A.; O'Sullivan, C.; O’Donnell, C.P. Terahertz time domain spectroscopy and imaging: Emerging techniques for food process monitoring and quality control. Trends Food Sci. Technol. 2012, 25, 40-46. [CrossRef]

16. Ogawa, Y.; Kondo, N.; House, G. Feasibility of using attenuated totalreflectance terahertz (ATR-THz) spectroscopy for quantitative and qualitativestudy of vitamin $C$ in aqueous solutions. In Proceedings of the ASABE Annual International Meeting, Louisville, KY, USA, 7-10 August 2011.

17. Nishikiori, R.; Yamaguchi, M.; Takano, K.; Enatsu, T.; Tani, M.; de Silva, U.C.; Kawashita, N.; Taragi, T.; Morimoto, S.; Hangyo, M.; et al. Application of partial least square on quantitative analysis of L-, D-, and DL-tartaric acid by terahertz absorption spectra. Chem. Pharm. Bull. 2008, 56, 305-307. [CrossRef] [PubMed]

18. Hua, Y.F.; Zhang, H.J. Qualitative and Quantitative Detection of Pesticides with Terahertz Time-Domain Spectroscopy. IEEE Trans. Microw. Theory 2010, 58, 2064-2070.

19. Baek, S.H.; Kang, J.H.; Hwang, Y.H.; Ok, K.M.; Kwak, K.; Chun, H.S. Detection of Methomyl, a Carbamate Insecticide, in Food Matrices Using Terahertz Time-Domain Spectroscopy. J. Infrared Millim. Terahertz Waves 2016, 37, 486-497. [CrossRef]

20. Qin, J.Y.; Xie, L.J.; Ying, Y.B. Feasibility of Terahertz Time-Domain Spectroscopy to Detect Tetracyclines Hydrochloride in Infant Milk Powder. Anal. Chem. 2014, 86, 11750-11757. [CrossRef] [PubMed]

21. Smith, G.; Hussain, A.; Bukhari, N.I.; Ermolina, I. Quantification of residual crystallinity in ball milled commercially sourced lactose monohydrate by thermo-analytical techniques and terahertz spectroscopy. Eur. J. Pharm. Biopharm. 2015, 92, 180-191. [CrossRef] [PubMed]

22. Sleiman, J.B.; Bousquet, B.; Palka, N.; Mounaix, P. Quantitative Analysis of Hexahydro-1,3,5-trinitro-1,3,5, Triazine/Pentaerythritol Tetranitrate (RDX-PETN) Mixtures by Terahertz Time Domain Spectroscopy. Appl. Spectrosc. 2015, 69, 1464-1471. [CrossRef] [PubMed]

23. Ge, H.Y.; Jiang, Y.Y.; Xu, Z.H.; Lian, F.Y.; Zhang, Y.; Xia, S.H. Identification of wheat quality using THz spectrum. Opt. Express 2014, 22, 12533-12544. [CrossRef] [PubMed]

24. Zhang, X.C.; Xu, J. Introduction to THz Wave Photonics; Springer: New York, NY, USA, 2009.

25. Dorney, T.D.; Baraniuk, R.G.; Mittleman, D.M. Material parameter estimation with terahertz time-domain spectroscopy. J. Opt. Soc. Am. A 2001, 18, 1562-1571. [CrossRef]

26. Shaddad, S.M.; Madrau, S.; Castrignano, A.; Mouazen, A.M. Data fusion techniques for delineation of site-specific management zones in a field in UK. Precis. Agric. 2016, 17, 200-217. [CrossRef]

27. Liang, X.Y.; Li, X.Y.; Lei, T.W.; Wang, W.; Gao, Y. Study of sample temperature compensation in the measurement of soil moisture content. Measurement 2011, 44, 2200-2204. [CrossRef]

28. Varshney, P.K. Distributed Detection and Data Fusion; Springer-Verlag: New York, NY, USA, 1996.

29. Roussel, S.; Bellon-Maurel, V.; Roger, J.-M.; Grenier, P. Fusion of aroma, FT-IR and UV sensor data based on the Bayesian inference. Application to the discrimination of white grape varieties. Chemom. Intell. Lab. 2003, 65, 209-219. [CrossRef]

30. Rudnitskaya, A.; Kirsanov, D.; Legin, A.; Beullens, K.; Lammertyn, J.; Nicolaï, B.M.; Irudayaraj, J. Analysis of apples varieties - Comparison of electronic tongue with different analytical techniques. Sens. Actuators B Chem. 2006, 116, 23-28. [CrossRef]

31. Cristianini, N.; Shawe-Taylor, J. An Introduction to Support Vector Machines and Other Kernel-Based Learning Methods; Cambridge University Press: Cambridge, UK, 2000.

32. Ge, H.Y.; Jiang, Y.Y.; Lian, F.Y.; Zhang, Y.; Xia, S.H. Quantitative determination of aflatoxin B1 concentration in acetonitrile by chemometric methods using terahertz spectroscopy. Food Chem. 2016, 209, 286-292. [CrossRef] [PubMed]

33. Dempster, A.P. Upper and lower probabilities induced by a multi-valued mapping. Ann. Math. Stat. 1967, 38, 325-339. [CrossRef]

34. Shafer, G. A Mathematical Theory of Evidence; Princeton University Press: Princeton, NJ, USA, 1976.

35. Wickramarathne, T.L.; Premaratne, K.; Murthi, M.N. Toward Efficient Computation of the Dempster-Shafer Belief Theoretic Conditionals. IEEE Trans. Cybern. 2013, 43, 712-724. [CrossRef] [PubMed]

36. Jiang, Y.Y.; Ge, H.Y.; Lian, F.Y.; Zhang, Y.; Xia, S.H. Early detection of germinated wheat grains using terahertz image and chemometrics. Sci. Rep. 2016, 6, 21299. [CrossRef] [PubMed]

37. Cortes, C.; Vapnik, V. Support-vector networks. Mach. Learn. 1995, 20, 273-297. [CrossRef] 
38. Chua, H.S.; Upadhya, P.C.; Haigh, A.D.; Obradovic, J.; Gibson, A.A.P.; Linfield, E.H. Terahertz time-domain spectroscopy of wheat grain. In Proceedings of the Conference Digest of the 2004 Joint 29th International Conference on Infrared and Millimeter Waves and 12th International Conference on Terahertz Electronics, Karlsruhe, Germany, 27 September-1 October 2004; pp. 399-400.

39. Guo, T.T.; Ding, Z.L.; Zhang, D.; Zhou, J.; Yang, Z.J.; Wang, X.L.; Yu, J.S.; Chen, X.D. Evaluation of wheat seeds by terahertz imaging. In Proceedings of the 2013 6th UK, Europe, China Millimeter Waves and THz Technology Workshop (UCMMT), Rome, Italy, 9-11 September 2013. [CrossRef]

(C) 2018 by the authors. Licensee MDPI, Basel, Switzerland. This article is an open access article distributed under the terms and conditions of the Creative Commons Attribution (CC BY) license (http:/ / creativecommons.org/licenses/by/4.0/). 